\section{Esophageal hematoma: a masquerade of rare occurrence}

A 72-year-old man who was receiving warfarin treatment for atrial fibrillation presented with hematemesis associated with retrosternal discomfort. He had swallowed a chicken bone 11 days before presentation. Hemodynamically, the patient was stable. The hemoglobin level was normal, but both the prothrombin time and the international normalized ratio were prolonged. Electrocardiography showed atrial fibrillation, with no ischemic changes. Endoscopy revealed an extensive longitudinal lesion with a bluish hue in the esophagus, extending from just beyond the upper esophageal sphincter to the gastroesophageal junction. It appeared as an elevated plaque bulging into the lumen of the esophagus (Figure 1). Contrast-enhanced computed tomography (CT) demonstrated a concentric mural thickening along the entire length of the thoracic esophagus (Figure 2). A diagnosis of esophageal hematoma was made. Warfarin treatment was stopped, and the patient was kept on a nothing-by-mouth regimen. A liquid diet was resumed 3 days later, and he was discharged free of symptoms after 9 days. The hematoma had regressed completely at endoscopy 8 weeks later (Figure 3).

Esophageal hematoma, a rare clinical entity, is part of the spectrum of esophageal injury. It can mimic other more serious or life-threatening conditions in various clinical aspects. The appearance at endoscopy can be mistaken for an esophageal malignancy or an aortoesophageal fistula from an aortic aneurysm [1,2]. Contrastenhanced CT is useful to exclude aortic pathologies [3]. However, the CT findings may be misinterpreted as a neoplasm by less experienced examiners [2]. Obtaining biopsy specimens from the lesion can lead to grave consequences, such as bleeding and perforation [4]. Misdiagnosis can occur when esophageal hematoma presents with acute chest pain. The acute onset and nature of the pain resemble that of aortic dissection, myocardial infarction, or pulmonary embolism $[2,4]$. Awareness and recognition of this entity is critical,

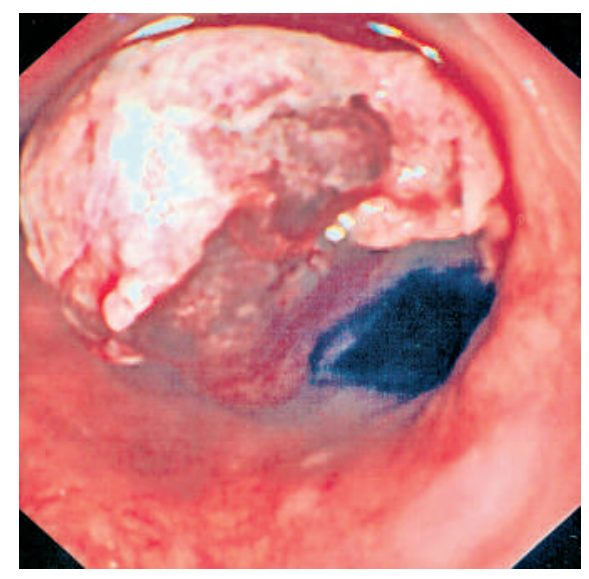

Figure 1 The endoscopic appearance of the hematoma at presentation.

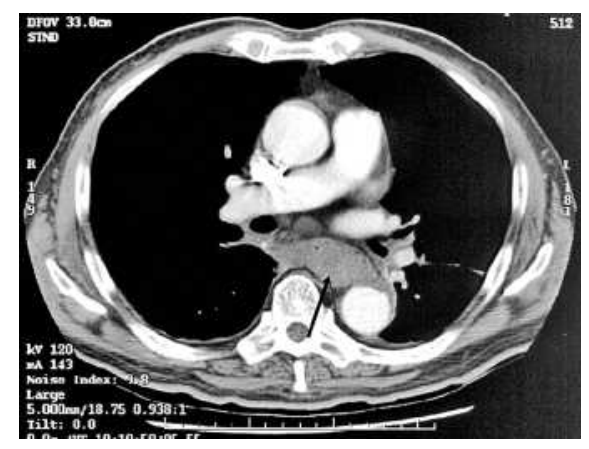

Figure 2 Contrast-enhanced computed tomography of the thorax demonstrates concentric and marked thickening of the esophageal wall (arrow). There is no aortic aneurysm or mediastinal collection.

because conservative treatment is the mainstay of management; further invasive investigations or treatments are potentially dangerous.

Endoscopy_UCTN_Code_CCL_1AB_2AC_3AG

\section{K.-L. Kwan, S. Law, K.-H. Wong, K.-F. Kwok \\ Division of Esophageal Surgery, \\ Dept. of Surgery, University of Hong Kong Medical Center, Queen Mary Hospital, Hong Kong.}

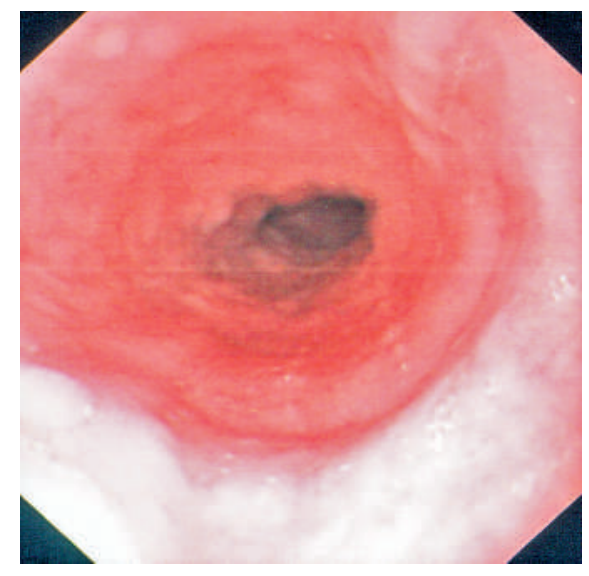

Figure 3 The endoscopic appearance 8 weeks later, showing complete resolution of the hematoma.

\section{References}

${ }^{1}$ Geller A, Gostout CJ. Esophagogastric hematoma mimicking a malignant neoplasm: clinical manifestations, diagnosis and treatment. Mayo Clin Proc 1998; 73: 342 - 345

2 Meulman N, Evans J, Watson A. Spontaneous intramural haematoma of the oesophagus: a report of three cases and review of the literature. Aust N Z J Surg 1994; 64: 190 - 193

${ }^{3}$ Herbetko J, Delany D, Ogilvie BC, Blaquiere RM. Spontaneous intramural haematoma of the oesophagus: appearance on computed tomography. Clin Radiol 1991; 44: 327-328 ${ }^{4}$ Skillington PD, Matar KS, Gardner MAH et al. Intramural haematoma of the oesophagus complicated by perforation. Aust N Z J Surg 1989; 59: $430-432$

\section{Corresponding author}

\section{S. Law, M.D.}

Dept. of Surgery

University of Hong Kong Medical Centre

Queen Mary Hospital

102 Pokfulam Road

Hong Kong

Fax: $\quad+852-2819-4221$

E-mail: slaw@hku.hk 